\title{
Chiral symmetry restoration in QCD with many flavours
}

\author{
Maria Paola Lombardo* \\ INFN-Laboratori Nazionali di Frascati, I-00044, Frascati (RM), and INFN-Sezione di Pisa, \\ I-56123, Pisa, Italy \\ E-mail: lombardo@lnf.infn.it
}

\section{Kohtaroh Miura}

Kobayashi-Maskawa Institute for the Origin of Particles and the Universe, Nagoya University, Nagoya 464-8602,Japan

E-mail: Kohtaroh.Miura@cpt.univ-mrs.fr

\section{Tiago J. Nunes da Silva, Elisabetta Pallante}

Centre for Theoretical Physics, University of Groningen, 9747 AG, Netherlands

E-mail: tiagoj.nunes@gmail.com, e.pallante@rug.nl

\begin{abstract}
We discuss the phases of QCD in the parameter space spanned by the number of light flavours and the temperature with respect to the realisation of chiral and conformal symmetries. The intriguing interplay of these symmetries is best studied by means of lattice simulations, and some selected results from our recent work are presented here.
\end{abstract}

9th International Workshop on Critical Point and Onset of Deconfinement

17-21 November, 2014

ZiF (Center of Interdisciplinary Research), University of Bielefeld, Germany

\footnotetext{
*Speaker.
} 


\section{Chiral symmetry and conformal symmetry in QCD with many flavours}

In this talk, at a variance with the phase diagram in the temperature, chemical potential plane much discussed at this meeting, we will explore the phases of QCD varying the temperature and the number of flavours. This is of course a vast subject[1], and here we will limit ourselves to the presentation of some of our recent results $[2,3]$.

In general, we know that framing a theory in a larger parameter space often adds to our understanding. More specifically, if the new axis is the number of light flavours, we have the chance to access a new regime of strong interactions: when the number of flavours exceeds a critical number, an infra-red fixed point (IRFP) appears and prevents the coupling from growing large enough to break chiral symmetry. The theory is then conformal invariant, and this remains true till asymptotic freedom is lost: the range of flavours between the onset of conformality and the loss of asymptotic freedom is known as the conformal window of strong interactions. Inside the conformal window the running of the coupling is regulated by two fixed point: the familiar UV fixed point at large momentum scales, and this novel IRFP for small momenta. Very interestingly, the coupling at the IRFP can still be largish: we can learn about strongly interacting conformal theories, and we can assess up to which extent such theories might offer a guidance to the physics of the strongly interactive quark gluon plasma.

Let us remind ourselves that (near)conformality plays quite an important role in the modelling of the plasma: bulk viscosity is set to zero by fiat in many hydrodynamics study, and in general AdS/CFT predictions have been widely scrutinised and contrasted with experimental observations and numerical results- a significant example of these predictions is the low value of the viscosity to entropy ratio, $\frac{\eta}{S} \simeq 1 / 4 \pi$, which appears to describe well the hydrodynamic evolution of the plasma after the collisions. To make more transparent a possible connection, let us then consider that both physics intuition and phenomenological analysis based on functional renormalisation group [4] and finite temperature holographic QCD [5]- [9] indicate that the conformal phase of cold, many flavour QCD and the high temperature chirally symmetric phase are continuously connected. In particular, the onset of the conformal window coincides with the vanishing of the transition temperature, and the conformal window appears as a zero temperature limit of a possibly strongly interacting QGP.

From a general field theory viewpoint, the analysis of the phase diagram of strong interactions as a function of the number of flavours adds to our knowledge of strong interactions and their fundamental mechanisms. From a particle phenomenology viewpoint, this study deals with a class of models which might play a relevant role in model building beyond the standard model, which explain the origin of mass using strong coupling mechanisms.

A simplified view of the phase diagram which we will consider here is sketched Figure 1: the axis is simply the number of light flavours. Ordinary QCD belongs to the hadronic phase. The conformal region is on the right hand side, and is separated by an essential singularity from the hadronic phase. Clearly, as in any system undergoing a phase transition, the nature and extent of the critical window are purely dynamical questions whose answer cannot be guessed a priori. Since the underlying dynamics is completely non-perturbative, lattice calculations are the only tool to perform an ab initio, rigorous study of these phenomena[12]-[46].

We now turn to the presentation of our results. The following Section is a concise summary 


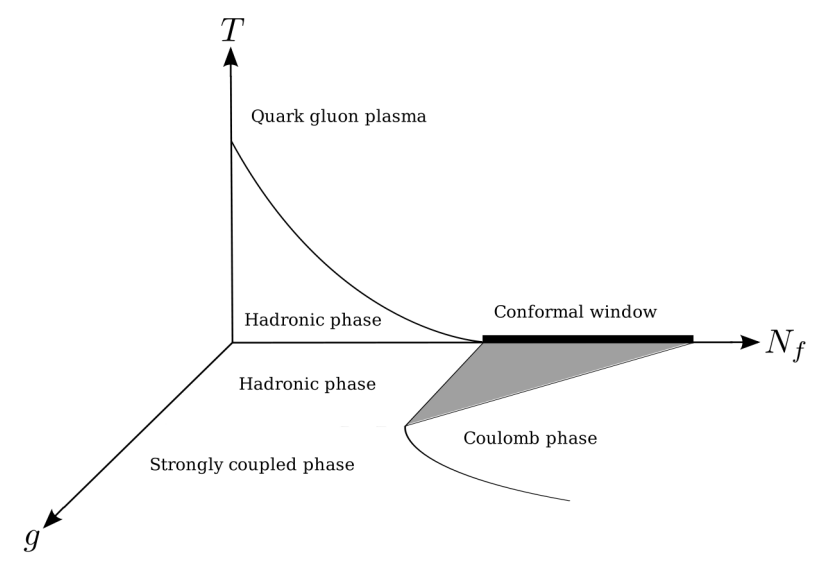

Figure 1: A sketch of the phase diagram of QCD-like theories in the temperature $(T)$, flavour number $\left(N_{f}\right)$ and bare coupling $(g)$ space. In the T- $N_{f}$ plane, the critical line is a phase boundary between the chirally broken hadronic phase and the chirally symmetric quark gluon plasma, the zero temperature end point of which is the onset of the conformal window. The zero temperature projected plane is inspired by the scenario in Refs. [47].

of our most recent paper Ref.[3] devoted to the analysis of the conformal phase for a fixed value of $N_{f}=12$. Then, following the presentation of our recent review Ref.[2], we concentrate on the preconformal phase, discussing first some of its general features, and then focussing on the strength of the coupling of the Quark Gluon Plasma as a function of $N_{f}$. We close with a brief summary.

\section{The cold conformal phase}

We study the $S U(3)$ gauge theory with twelve flavours of fermions in the fundamental representation as a possible realisation of a conformal theory. Following the phase diagram sketched in Figure 1, we consider the Coulomb phase which appears for lattice couplings larger than the one where the beta function vanishes. Our main result here is to show that the spectrum in the Coulomb phase of the system can be described in the context of a universal conformal scaling analysis. We then provide the nonperturbative determination of the fermion mass anomalous dimension $\gamma^{*}=0.235(46)$ at the infrared fixed point. Our recent work discusses in detail how the ideal conformal scaling law should be modified by finite volume and other lattice artefacts. The remarkable outcome is that all the results from different groups indicate the same anomalous dimension once such corrections are properly taken into account.

Figure 2 summarises this study, where we show the collapse on a common universal curve of data obtained with different lattice actions and lattice couplings, once perturbative corrections to the universal scaling are divided out. Details on the fits and optimal parameters can be found in Ref.[2].

This analysis leads to the determination of the mass anomalous dimension $\gamma^{*}$ at the IRFP. We quote the value

$$
\delta=0.81(3) \quad \gamma^{*}=0.235(46)
$$

This value is in agreement with the perturbative four-loop prediction, with the best-fit result of [26] and not far from the first lattice determination of the fermion mass anomalous dimension for the 


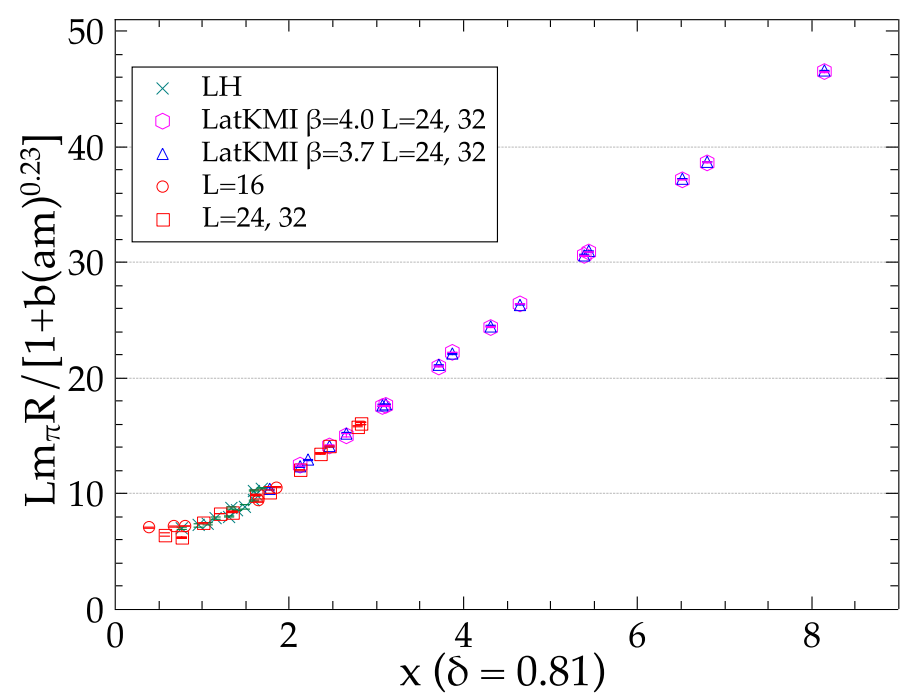

Figure 2: Collapse of curves for the rescaled pseudoscalar product $L m_{\pi} R /\left(1+b m^{\omega}\right)$, with $\omega=0.23$ from 4-loop perturbation theory as a function of the universal scaling variable $x=L \mathrm{~m}^{\delta}$ with $\delta=0.81$ from this work. Data are from [32] at $\beta=2.2$ (LH, green crosses), [24] at $\beta=4.0$ (LatKMI, magenta hexagons) and $\beta=3.7$ (LatKMI, blue triangles), and from this work for $L=24,32$ (red squares) and $L=16$ (red circles). From Ref. [2].

$N_{f}=12$ system in [17], though the latter was affected by rather large uncertainties.

To summarise, a universal power law with exponent $\delta=0.81$ describes all would-be hadrons, with additional perturbative mass corrections of the type $1+\Delta g m^{\delta \gamma_{s}^{*}}$ in the vector and axial channels. The pseudoscalar is the lightest state, but it is not a Goldstone boson. The vector, the scalar, the axial, and finally the nucleon follow.

The identification of universal contributions dictated by the conformal invariance at the fixed point and deviations from universal scaling induced in the surroundings of the fixed point has allowed for the nonperturbative determination of the fermion mass anomalous dimension $\gamma^{*}=$ 0.235 (46) and a unified description of all lattice results for the would-be hadron spectrum of the $N_{f}=12$ theory.

\section{Near-conformal: exploring scales}

In this Section we discuss results for $N_{f}=0,4,6,8$, approaching the conformal window from below. In this case the results have been obtained with a fixed bare quark mass, and no attempt has been done to extrapolate to the chiral limit. Obviously in this case all the notions of symmetries are approximate, due the explicit mass (chiral breaking) term.

In order to monitor the behaviour of these theories we had to choose some observables: we have considered the (pseudo)critical temperature, the 'most UV scale' available on the lattice (which will be explained later), the zero temperature string tension, and the $w_{0}$ scale[48] defined by the Wilson flow [49] (the preliminary results for this latter observables will not be shown here). 


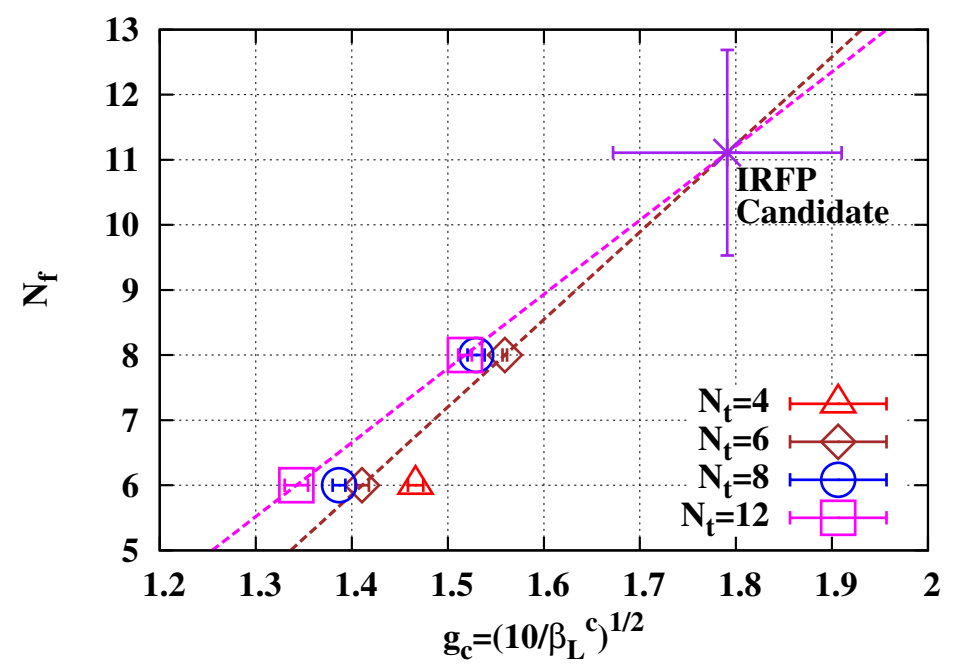

Figure 3: (Pseudo) critical values of the lattice coupling $g_{\mathrm{L}}^{\mathrm{c}}=\sqrt{10 / \beta_{\mathrm{L}}^{\mathrm{c}}}$ for theories with $N_{f}=6,8$ and for several values of $N_{t}$ in the Miransky-Yamawaki phase diagram. We have picked up $g_{\mathrm{L}}^{\mathrm{c}}$ at $N_{f}=6$ and 8 , and considered "constant $N_{t}$ " lines with $N_{t}=6,12$. If the system is still described by one parameter beta-function in this range of coupling, the IRFP could be located at the intersection of the fixed $N_{t}$ lines or equivalently, in the region where the step-scaling function vanishes. To demonstrate the procedure -as a preliminary example - we have considered the intersection of the $N_{t}=12$ and $N_{t}=6$ lines

\section{1 (Pseudo)critical temperature}

We consider first the (pseudo)critical lattice couplings $\beta_{\mathrm{L}}{ }^{\mathrm{c}}$ as a function of $N_{f}$ and $N_{t}$ associated with the thermal crossover which can be observed also with a finite mass. Let us plot $g_{\mathrm{L}}^{\mathrm{c}}\left(N_{f}, N_{t}\right)=$ $\sqrt{10 / \beta_{\mathrm{L}}^{\mathrm{c}}\left(N_{f}, N_{t}\right)}$ in the space spanned by the bare coupling $g_{\mathrm{L}}$ and the number of flavour $N_{f}$, and consider the lines which connect $g_{\mathrm{L}}^{\mathrm{c}}$ with $N_{t}$ fixed: $\left.g_{\mathrm{L}}^{\mathrm{c}}\left(N_{f}\right)\right|_{N_{t}=\text { fix }}$ (see Fig. 3). We consider the pseudo-critical lines obtained at fixed $N_{t}$ : the critical number of flavour $N_{f}^{*}$ can be read off from their crossing point - simply because this crossing point correspond to a zero of the lattice beta function. In Fig. 3 we show the pseudo-critical lines obtained for $N_{t}=6$ and $N_{t}=12$ whose intersection can be estimated at $\left(g_{\mathrm{L}}^{\mathrm{c}}, N_{f}^{*}\right)=(1.79 \pm 0.12,11.1 \pm 1.6)$. We emphasise that this is a finite mass results, implying that $N_{f}^{*}$ is an upper limit to the chiral limit critical number of flavours.

Let us now fix $N_{f}$ and consider the pseudo-critical temperatures $T_{c}$ in physical units:

$$
T_{c} \equiv \frac{1}{a\left(\beta_{\mathrm{L}}^{\mathrm{c}}\right) \cdot N_{t}} .
$$

We introduce the normalised critical temperature $T_{c} / \Lambda_{\mathrm{L} / \mathrm{E}}$ (see e.g. [50]) where $\Lambda_{\mathrm{L}}\left(\Lambda_{\mathrm{E}}\right)$ represents the lattice (E-scheme) Lambda-parameter defined in the two-loop perturbation theory with or without a renormalisation group inspired improvement [51]. We have observed that $T_{C} / \Lambda$, regardless the prescription used, approaches a constant by increasing $N_{t}$, enabling us (with the due caveats) to interpret these asymptotic values as continuum estimates. We can then consider their $N_{f}$ dependence : $T_{c} / \Lambda$ apparently increases with $N_{f}$. This suggests that $\Lambda$ vanishes faster than $T_{c}$ when approaching $N_{f}^{*}$, i.e. has a strong sensitivity to the IR dynamics affected by the conformal threshold. 


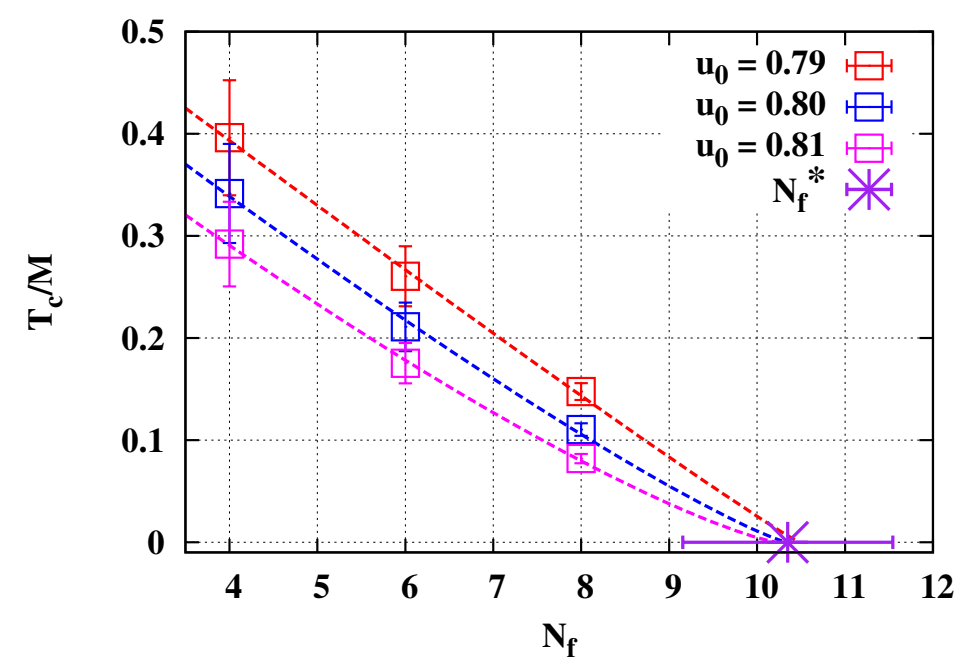

Figure 4: The $N_{f}$ dependence of $T_{c} / M$ where $M$ is determined to be a UV scale corresponding to different, equivalent, prescriptions and $T_{c}$ is the pseudocritical temperature for a bare quark mass $m_{q}=0.02$.

We would like now to define a UV reference scale for different $N_{f}$. We have chosen a perturbative value for the coupling and identified - with the help of two loop scaling - a corresponding mass scale which can be then used to normalise $T_{c}$ for different temperatures. The outcome of this procedure is demonstrated in Figure 4. On such UV scale $T_{c}$ is now a decreasing function of $N_{f}$, as expected. Since these results have been obtained at a finite mass, the critical number of flavours in the chiral limit is encoded in the appropriate generalisation of the magnetic equation of state, and at least another mass value would be needed to fix its free parameters (the normalizations). A semiqualitative extrapolation of the data suggest that $T_{c}\left(m_{q}\right)$ would equal zero for $N_{f}^{*}=10.4 \pm 1.2$. Again this finite mass estimate provides an upper limit to the critical number of flavours in the chiral limit. A similar reasoning brings to the same conclusion in the case of a first order transition. We emphasise that the logic of this exercise is not to find a precise estimate of the critical number of flavours, rather to provide an evidence that such a critical number of flavours does exist, and its approach can be observed. If and where the critical temperature in the chiral limit reaches zero is a question obviously beyond the scope of an analysis performed with a fixed value of the quark mass.

Next, we have measured the zero temperature string tension $\sigma$ from Wilson Loops. $\sigma$ has been evaluated for the same set of pseudocritical couplings we have identified in our thermal study. Because of this, we can immediately compute $T_{c} / \sqrt{\sigma}$ (Figure 5): we note that the decreasing trend becomes less apparent with increasing $N_{f}$. Interestingly, this behaviour compares nicely with the findings of a recent holographic study [9].

\section{Which is the strongest coupled QGP?}

We consider here the coupling $g_{\mathrm{T}}^{\mathrm{c}}\left(N_{f}\right)$ at the scale of the (pseudo)critical temperature for each $N_{f}$ - having established with reasonable confidence asymptotic scaling we use perturbation theory 


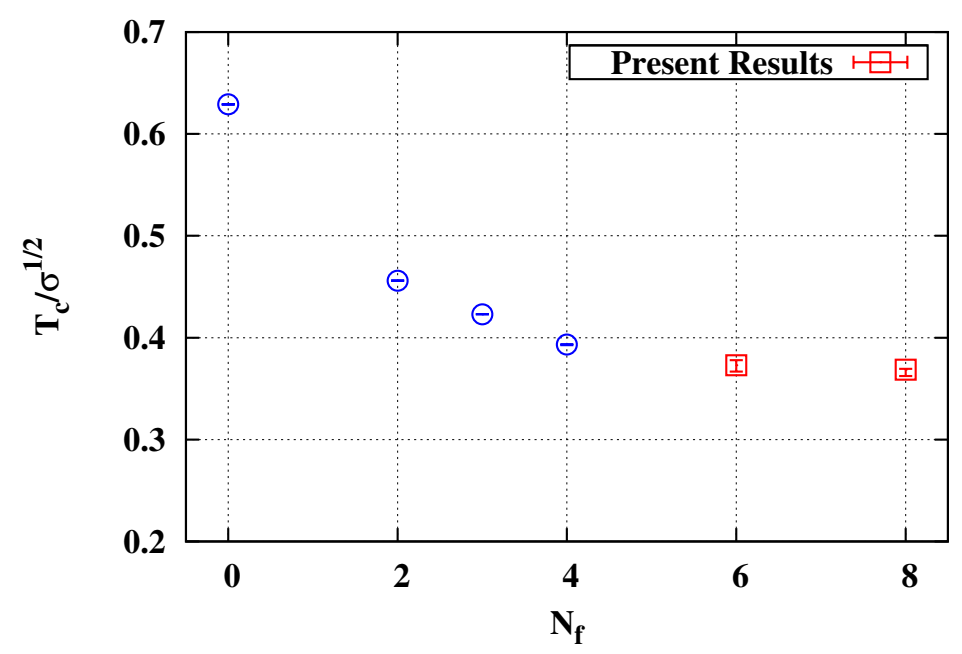

Figure 5: The $T_{c} / \sqrt{\sigma}$ as a function of $N_{f}$. The symbol $\square$ (red) represents the present results $\left(N_{f}=6,8\right)$. For a comparison, we have quoted the $T_{c} / \sqrt{\sigma}$ from [52] $\left(N_{f}=0\right)$, [53] $\left(N_{f}=2,3\right)$, [54] $\left(N_{f}=4\right)$, shown as $\bigcirc$ (blue) symbols. These results are still preliminary.

to evolve the coupling at the scale of the lattice spacing $a$ up to the temperature inverse scale $N_{t} a$.

The red ( $\square$ ) symbol in Figure 6 shows $g_{\mathrm{T}}^{\mathrm{c}}$ as a function of $N_{f}$. We superimpose a fit obtained by using the ansatz proposed in Ref. [55]

$$
N_{f}\left(g_{\mathrm{T}}^{\mathrm{c}}\right)=A \cdot \log \left[B \cdot\left(g_{\mathrm{T}}^{\mathrm{c}}-\left.g_{\mathrm{T}}^{\mathrm{c}}\right|_{N_{f}=0}\right)+1\right]
$$

with $A$ and $B$ fit parameters, which describes well the data. We note that $g_{\mathrm{T}}^{\mathrm{c}}$ is an increasing function of $N_{f}$. This indicates that the quark-gluon plasma is more strongly coupled at larger $N_{f}$, as discussed in Ref. [55]. In turn, this observation might provide a clue into the nature of the strongly interactive quark gluon plasma.

\section{Summary}

We have described some studies whose ultimate aim is a quantitative characterisation of the phase diagram in Figure 1. We briefly summarise here a few results: the pseudocritical temperature $T_{c}$ as a function of the number of light flavours, once properly normalised by use of a UV scale, is a decreasing function of $N_{f}$. The results on $T_{c} / \sqrt{\sigma}$ show a comparably milder decrease. These observations are compatible with an (essential?) critical behaviour and scale separation in the precritical region. The conformal phase is strongly interactive with anomalous dimension, and the (strongly coupled) Quark Gluon Plasma is smoothly connected with such cold conformal strongly interacting phase. The latter observation might offer some motivation and guidance for studies based on AdS/CFT correspondence and related holographic approaches. 


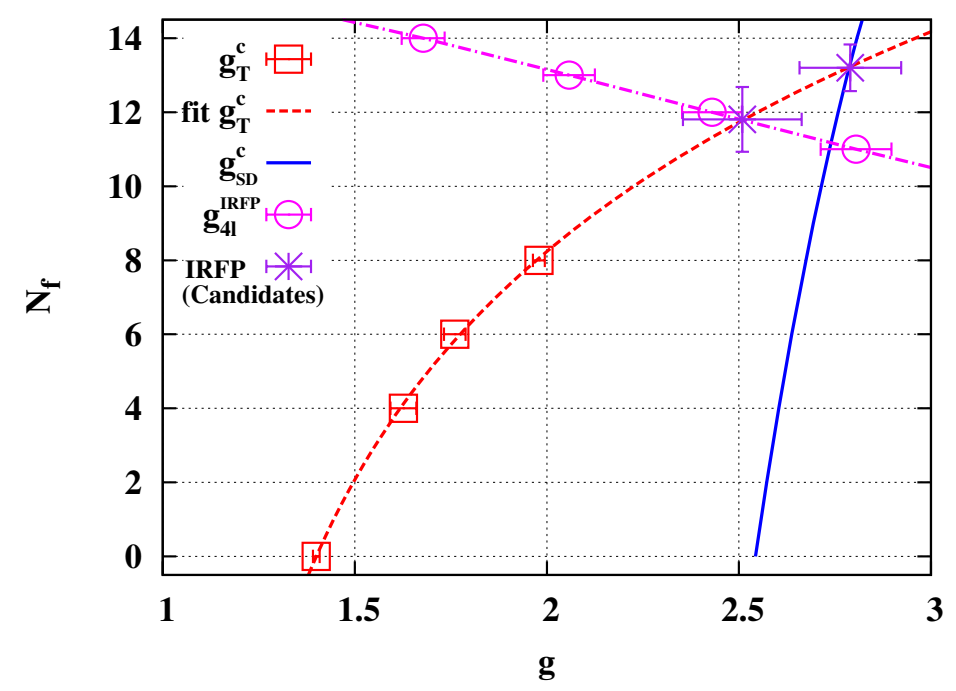

Figure 6: The couplings at $T_{c}$ (red $\square$ ) and the fit for them (dashed red line, with the ansatz Eq. (4.1)) and the values of the zero temperature couplings in the conformal phase from different analytic estimates. The thermal coupling increases with $N_{f}$ and at the critical number of flavours it should equal the coupling associated with the IRFP.

\section{Acknowledgments}

MpL wishes to thank the organisers of CPOD2014 for a very interesting meeting and a warm hospitality in Bielefeld. In addition, the support of the Galileo Galilei Institute during the workshop 'Holographic Methods for Strongly Coupled Systems' is gratefully acknowledged. We wish to thank Marc Wagner for providing us the code for the Wilson loop measurements. The numerical calculations were performed at CINECA in Italy, at YITP, Kyoto University in Japan, and at KMI, Nagoya University in Japan. This work was in part based on the MILC Collaboration's public lattice gauge theory code [56].

\section{References}

[1] The reader might want to consult the proceedings of the conferences Strong Coupling Gauge Theories, Nagoya, 2009, 2012 and 2015 (upcoming) for comprehensive background material.

[2] M. P. Lombardo, K. Miura, T. J. Nunes da Silva and E. Pallante, Int. J. Mod. Phys. A 29 (2014) 25.

[3] M. P. Lombardo, K. Miura, T. J. Nunes da Silva and E. Pallante, JHEP 1412 (2014) 183.

[4] J. Braun, C. S. Fisher, H. Gies, Phys. Rev. D84 (2011) 034045.

[5] M. Järvinen and E. Kiritsis, JHEP 1203 (2012) 002.

[6] T. Alho, M. Järvinen, K. Kajantie, E. Kiritsis and K. Tuominen, JHEP 1301 (2013) 093.

[7] U. Gursoy, E. Kiritsis, L. Mazzanti, G. Michalogiorgakis and F. Nitti, Lect. Notes Phys. 828 (2011) 79.

[8] T. Alho, N. Evans and K. Tuominen, Phys. Rev. D 88 (2013) 105016. 
[9] F. Bigazzi and A. L. Cotrone, JHEP 1501 (2015) 104.

[10] J. B. Kogut, J. Polonyi, H. W. Wyld and D. K. Sinclair, Phys. Rev. Lett. 54, 1475 (1985).

[11] P. H. Damgaard, U. M. Heller, A. Krasnitz and P. Olesen, Phys. Lett. B 400 (1997) 169.

[12] T. Appelquist, G. T. Fleming, M. F. Lin, E. T. Neil, D. A. Schaich, Phys. Rev. D84, 054501 (2011).

[13] T. Appelquist, G. T. Fleming and E. T. Neil, Phys. Rev. D 79 (2009) 076010.

[14] T. Appelquist et al., Phys. Rev. Lett. 104 (2010) 071601.

[15] T. Appelquist et al. [ LSD Collaboration ], Phys. Rev. Lett. 106 (2011) 231601.

[16] A. Deuzeman, M. P. Lombardo and E. Pallante, Phys. Lett. B 670 (2008) 41.

[17] A. Deuzeman, M. P. Lombardo and E. Pallante, Phys. Rev. D 82 (2010) 074503.

[18] A. Deuzeman, M. P. Lombardo, T. Nunes Da Silva and E. Pallante, Phys. Lett. B 720 (2013) 358.

[19] K. Miura, M. P. Lombardo and E. Pallante, Phys. Lett. B 710 (2012) 676.

[20] K. Miura and M. P. Lombardo, Nuclear Physics B 871 (2013) 52.

[21] Y. Aoki et al. [the LatKMI Collaboration], Phys. Rev. D 89 (2014) 111502.

[22] Y. Aoki et al. [LatKMI Collaboration], Phys. Rev. Lett. 111 (2013) 16, 162001.

[23] Y. Aoki et al. [LatKMI Collaboration], Phys. Rev. D 87 (2013) 9, 094511.

[24] Y. Aoki, T. Aoyama, M. Kurachi, T. Maskawa, K. -i. Nagai, H. Ohki, A. Shibata and K. Yamawaki et al., Phys. Rev. D 86 (2012) 054506.

[25] A. Cheng, A. Hasenfratz, Y. Liu, G. Petropoulos and D. Schaich, JHEP 1405 (2014) 137.

[26] A. Cheng, A. Hasenfratz, Y.Liu, G. Petropoulos and D. Schaich, Phys. Rev. D 90 (2014) 014509.

[27] A. Cheng, A. Hasenfratz, G. Petropoulos and D. Schaich, JHEP 1307 (2013) 061.

[28] A. Cheng, A. Hasenfratz and D. Schaich, Phys. Rev. D 85 (2012) 094509.

[29] A. Hasenfratz, Phys. Rev. Lett. 108 (2012) 061601.

[30] A. Hasenfratz, Phys. Rev. D 82 (2010) 014506.

[31] A. Hasenfratz, Phys. Rev. D 80 (2009) 034505.

[32] Z. Fodor, K. Holland, J. Kuti, D. Nogradi and C. Schroeder, Phys. Lett. B703 (2011) 348-358.

[33] Z. Fodor, K. Holland, J. Kuti, D. Nogradi and C. Schroeder, Phys. Lett. B 681 (2009) 353.

[34] K. -I. Ishikawa, Y. Iwasaki, Y. Nakayama and T. Yoshie, Phys. Rev. D 89 (2014) 114503.

[35] K. -I. Ishikawa, Y. Iwasaki, Y. Nakayama and T. Yoshie, Phys. Rev. D 87 (2013) 7, 071503.

[36] Y. Iwasaki, K. Kanaya, S. Kaya, S. Sakai and T. Yoshie, Phys. Rev. D 69 (2004) 014507.

[37] T. Karavirta, A. Mykkanen, J. Rantaharju, K. Rummukainen and K. Tuominen, JHEP 1106 (2011) 061.

[38] Y. Shamir, B. Svetitsky and E. Yurkovsky, Phys. Rev. D 83 (2011) 097502.

[39] J. B. Kogut and D. K. Sinclair, Phys. Rev. D 81 (2010) 114507.

[40] Z. Fodor, K. Holland, J. Kuti, D. Nogradi and C. Schroeder, JHEP 0911 (2009) 103. 
[41] Z. Fodor, K. Holland, J. Kuti, D. Nogradi, C. Schroeder and C. H. Wong, Phys. Lett. B 718 (2012) 657.

[42] S. Catterall, J. Giedt, F. Sannino and J. Schneible, JHEP 0811 (2008) 009.

[43] L. Del Debbio, B. Lucini, A. Patella, C. Pica and A. Rago, Phys. Rev. D 82 (2010) 014510.

[44] L. Del Debbio and R. Zwicky, Phys. Lett. B 700 (2011) 217.

[45] L. Del Debbio and R. Zwicky, Phys. Rev. D 82 (2010) 014502.

[46] Ph. de Forcrand, S. Kim and W. Unger, JHEP 1302 (2013) 051.

[47] V. A. Miransky and K. Yamawaki, Phys. Rev. D 55 (1997) 5051 [Erratum-ibid. D 56 (1997) 3768].

[48] S. Borsanyi, S. Durr, Z. Fodor, C. Hoelbling, S. D. Katz, S. Krieg, T. Kurth and L. Lellouch et al., JHEP 1209 (2012) 010.

[49] M. Luscher, Commun. Math. Phys. 293 (2010) 899.

[50] S. Gupta, Phys. Rev. D 64 (2001) 034507.

[51] C. R. Allton, Nucl. Phys. Proc. Suppl. 53 (1997) 867.

[52] E. Laermann, Nucl. Phys. A 610 (1996) 1C.

[53] F. Karsch, E. Laermann and A. Peikert, Nucl. Phys. B 605 (2001) 579.

[54] J. Engels, R. Joswig, F. Karsch, E. Laermann, M. Lutgemeier and B. Petersson, Phys. Lett. B 396 (1997) 210.

[55] J. Liao, E. Shuryak and E. Shuryak, Phys. Rev. Lett. 109, 152001 (2012).

[56] MILC Collaboration, http://www.physics.indiana.edu/sg/milc.html. 